\title{
Los congresos en la actual sociedad del conocimiento
}

\author{
Clara Janneth SANTOS MARTíNEZ \\ Universidad Autónoma de Bucaramanga (Colombia) \\ janneths@hotmail.com
}

Recibido: 30/11/2012

Aceptado: 23/01/2013

\section{Resumen}

El ámbito universitario esta llamado, hoy en día, a propiciar una verdadera cultura científica y promover la alfabetización científica y tecnológica, siguiendo los lineamientos del Espacio Europeo de Educación Superior y favoreciendo el acercamiento de los agentes de la educación a la Sociedad del Conocimiento. La comunicación científica expresada a través de los medios masivos, pero, principalmente, a través de publicaciones, congresos, jornadas, seminarios, etc., está viviendo los cambios propios de la Web 2.0, que crea nuevos roles y funcionalidades. Los congresos como eventos de comunicación y socialización de los resultados de investigación participan de este proceso transformador poniendo en marcha diversas experiencias innovadoras, entre las que las redes sociales instituyen una senda que conduce a nuevas tendencias como el Cloud Computing o servicio en la nube.

Palabras Clave: Congresos on-line; Sociedad del conocimiento; Web 2.0; Comunicación Científica

\section{Conferences in Today Knowledge Society}

\begin{abstract}
Today, Universities are called to create a true scientific culture and raise awareness about the need for scientific and technological literacy, in accordance with the guidelines of the European Space of Higher Education, and favoring a rapprochement of education agents to Knowledge Society. Scientific Communication expressed mainly through publications, conferences, workshops, seminars, and so, is undergoing the changes that the Web 2.0 is experiencing, and is thus creating new roles and functions. Congresses, as media events and a way of socialization of research findings- are involved in this process of transformation by putting into practice a series of innovative experiences, including social networking, which institute a path that leads to new trends such as Cloud Computing.

Keywords: On-line conferences; Knowledge Society; Web 2.0; Social Communication

\section{Referencia normalizada}

SANTOS MARTÍNEZ, Clara Janneth (2013): “Los congresos en la actual sociedad del conocimiento". Estudios sobre el mensaje periodístico. Vol. 19. Núm. especial abril, págs.: 995-1005. Madrid, Servicio de Publicaciones de la Universidad Complutense.
\end{abstract}

Sumario: 1. Introducción. 2. Metodología. 3. Desarrollo. 4. Antecedentes de experiencia congresual. 5. CUICIID virtual y en línea. 6. Conclusiones. 7. Referencias bibliográficas.

\section{Introducción}

Este trabajo compromete su interés científico en el afán de recoger algunas de las aportaciones que el uso de las TICS puede hacer a la educación y la ciencia, a fin de transmitirlas a la sociedad, yendo de lo particular a lo general y teniendo en consideración que, "Internet, proporciona un medio nuevo para obtener conocimientos y significados" (Negroponte, 1995: 239). El investigador que produce conocimientos se ve en la encrucijada de difundir dicho conocimiento, de acercarlo a la comunidad científica, de propiciar el debate científico, pero también de explicarlo a la sociedad, justificando en qué medida la ciencia parte de la realidad para construirla. 
Los congresos ${ }^{1}$-considerados en sentido amplio, jornadas, seminarios, mesas redondas, etc-, constituyen uno de los recursos más utilizados por científicos e investigadores para difundir los resultados de sus investigaciones acercando, a través del debate y la difusión, la ciencia a la sociedad. Además de ser reuniones en las que se brinda información (generalmente novedosa, mediante comunicaciones y/o ponencias individuales o grupales), en los congresos se destaca un aspecto comunicativo singular: en ellos el público asistente puede interactuar mediante réplicas y/o preguntas sobre los temas que se discuten, generándose una retroalimentación que determina roles para aquellos que asisten y/o participan en los congresos. Desde el punto de vista de la audiencia se consideran los siguientes roles en un congreso ${ }^{2}$ : Rol de autor (el asistente difunde los resultados de su investigación), rol de aprendizaje (el asistente recibe y aprende conocimientos bibliográficos, teóricos o metodológicos), rol de relación (el asistente mejora contactos, crea equipos interuniversitarios $\mathrm{y} / \mathrm{o}$ asiste al congreso como alternativa lúdica y cultural). (Ruiz-Santos, et al., 2007, web)

El presente artículo propone describir la forma como un congreso científico universitario, de carácter internacional, mediado por las Tecnologías de la Información y la Comunicación, TIC, además de proponer una estrategia de difusión de resultados de investigación en el área de comunicación, aprovechó las ventajas de la Web 2.0 innovando en su uso, a un coste que podría considerarse "cero", consiguiendo mayor visibilidad e impacto mediático para sus participantes $\mathrm{y}$, finalmente, propiciando un verdadero acercamiento del conocimiento científico a la sociedad, objetivo que, entre otros, la Unión Europea acoge bajo la política Science in Society, SIS ${ }^{3}$.

\section{Metodología}

Tomando la investigación cualitativa como marco metodológico para el estudio de un congreso on-line, en tanto que evento al servicio de la difusión del conocimiento científico y promotor del acercamiento ciencia - sociedad mediante la comunicación, se plantea entonces una reflexión que, mediante el estudio de caso, la observación participativa, y la entrevista en profundidad, como instrumentos de la investigación social, se analice y describa en qué medida, en la actual Sociedad de la Información, los congresos on-line son una potente herramienta de comunicación masiva, en los que, gracias al empleo de la web social, se generan nuevos roles y funcionalidades del trabajo científico e investigador, así como una mayor visibilidad, intercambio y cooperación para el trabajo de investigación.

1 Un Congreso se define por su realización periódica y por su carácter de reunión convocada para la discusión de temas concretos. DRAE.

2 Ruiz-Santos y Meroño-Cerdán presentan esta clasificación en su análisis factorial sobre la utilidad de los congresos.

3 El Programa Ciencia en la Sociedad comprende diversas perspectivas del compromiso con la sociedad, como por ejemplo, propiciar el diálogo entre científicos y otros miembros de la comunidad, mantener lazos de investigación que conecten ciencia, democracia y ley, ciencia ética sobre ciencia y la tecnología, reciprocidad entre ciencia y cultura, el papel y la imagen de los científicos, aspectos de género, métodos de ciencia y educación y comunicación científica. 


\section{Desarrollo}

La idea de reforzar, en Europa, los tres vértices del "triángulo del conocimiento": educación, investigación e innovación está directamente relacionada con el papel que juega la universidad y con los roles que profesores, investigadores, alumnos y personal de administración y servicios deben asumir ya que: "El crecimiento de la Sociedad del Conocimiento depende de la producción de nuevos conocimientos, su transmisión a través de la educación y la formación, su divulgación a través de las tecnologías de la información y la comunicación y su empleo por medio de nuevos procedimientos industriales o servicios" (Comisión, 2003, web)

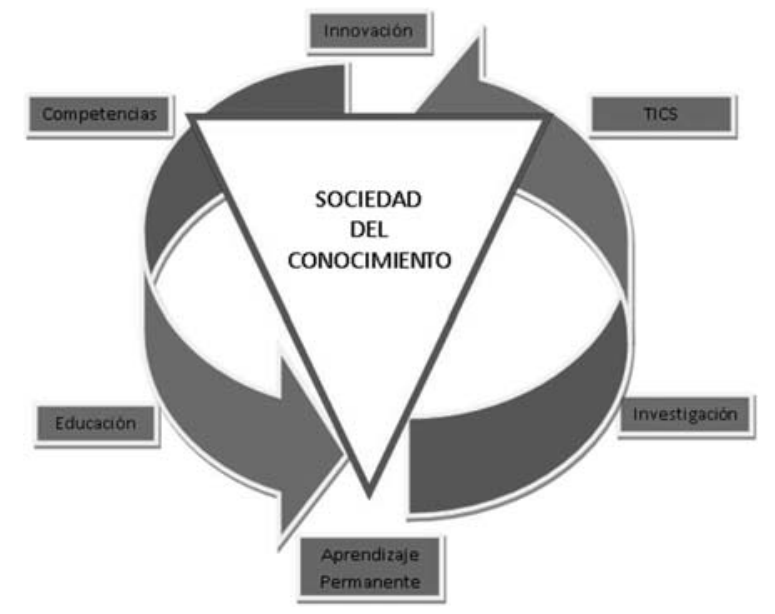

Figura 1. Elaboración propia. El triángulo del conocimiento Innovación-Investigación-Educación, requiere ciudadanos que manejen las TIC y adquieran las competencias propias para el Aprendizaje Permanente.

Publicaciones y congresos científicos han constituido por mucho tiempo las principales acciones mediante las cuales se difunde y socializa el trabajo científico o se proponen nuevos proyectos; sin embargo, durante las últimas décadas han aparecido nuevos modos de almacenamiento (CD's, memoria flash, Dropbox, Skydrive, etc), de publicación (Blogger, Wordpress, etc) y de difusión (Facebook, Twitter, Linkedin, Google Plus, etc) de los resultados de investigación que definen la preponderancia del escenario digital y la creación de nuevas representaciones y nuevos roles que determinan la forma como los investigadores dan a conocer los resultados de sus investigaciones (Torres-Salinas, et al., 2009, web), ya no basta con la publicación en las revistas convencionales sino que se acude a múltiples recursos en la web 2.0 (blogs, microblogs, redes sociales, alojamiento de presentaciones, repositorios temáticos), haciendo de este entramado un escenario experimental de múltiples aplicaciones y usos que enriquecen las posibilidades de almacenamiento, publicación y difusión de los resultados de investigación.

El acercamiento de la ciencia a la sociedad conlleva aumentar los canales de comunicación generando en el investigador la necesidad de realizar un verdadero marketing para aumentar la visibilidad e impacto de su investigación, acudiendo a canales 
convencionales y no convencionales de difusión. Las publicaciones científicas constituyen un tipo de literatura que tradicionalmente tiene como objeto la difusión del trabajo científico y que cada vez más, ocupa y preocupa a docentes investigadores en su afán por la acreditación profesional, prueba de ello son la creación de organismos que normalizan y dan cuenta de la forma como se evalúa la calidad de la formación y la investigación que se realiza en las universidades, y los estudios que, por ejemplo, dan cuenta de estrategias y formas de competir en esta dirección ${ }^{4}$.

Como se mencionó, actualmente surgen otros ámbitos para la difusión del conocimiento científico a través de Internet que presentan nuevas formas y roles para el trabajo del investigador que interactúa con las TIC, que generan una verdadera transformación de la comunicación científica (Hurd; 2000, web). Éstos son: formas en las que no se emplea el papel impreso, disminuye la preferencia por el artículo o publicación científica, manteniendo el sistema peer-review; en ellos no importa el lugar en el que el investigador se encuentre, ni la hora, puesto que la tecnología informática conecta y apoya colaboradores y organizaciones dispersas, se potencia el trabajo colaborativo e interdisciplinar, el intercambio o el análisis de los resultados de investigación en tiempo real, se incrementa el uso de los servicios de agregados, se interactúa mediante el uso del correo electrónico o el intercambio cara a cara mediante videoconferencias o encuentros síncronos o asíncronos, entre otros.

Pero, desde el punto de vista del acercamiento de la ciencia a la sociedad, los congresos representan una actividad socializadora más amplia, impacto mediático y, verdadero contacto (networking) gracias a su carácter de encuentro, de debate científico $\mathrm{y}$ al hecho de que las ponencias suelen ser material de base para futuros artículos. Se describe y analiza a continuación la realización de un congreso universitario internacional que se enmarca en el camino de estas transformaciones e innova en su modalidad de realización, empleando la red social Google Plus, difundiendo las ponencias on-line en .pdf, .mp3, avi, entre ellos, interactuando en multiconferencias y difundiendo su realización por vídeo, a través de You Tube, y dejando canales abiertos a la interactuación en directo y en diferido, con públicos potenciales.

\section{Antecedentes de experiencia congresual}

Este trabajo aporta ideas de un estudio de caso sobre la experiencia congresual en modalidad blended (virtual y presencial), realizada en 2010 y en la que ésta autora participó. (Santos, 2012:281). En ella se revisó el caso específico de la Universidad Autónoma de Bucaramanga, en Colombia, cuya experiencia congresual se virtualizó a través de la plataforma tecnológica Moodle desde la que, un grupo de 35 docentes, siguió el evento on-line y en diferido desde un aula virtual, intercambiando opiniones con un moderador a través del foro, el chat y el e-mail, adecuados a tal propósito. Moodle es un Sistema de Gestión de Aprendizaje o Learning Management System, LMS, orientado al E-Learning que se empleó para el seguimiento del Congreso desde el aula

4 En España, siguiendo los principios de Berlín para Rankings de Instituciones de Educación Superior, se elabora y publica, anualmente, desde 2009 el "Ranking de producción y productividad en investigación de las universidades públicas españolas” (Buela-Casal; Bermúdez; Sierra; Quevedo-Blasco; Castro; Guillén-Riquelme). 
virtual. Los participantes se matricularon a un curso en el que interactuaron entre sí, descargaron información y constituyeron una comunidad virtual síncrona y asíncrona en torno a los congresos realizados en Madrid y transmitidos por el canal UNED instalado en la web.

En 2010 la funcionabilidad de esta infraestructura web a través del aula virtual, conectando usuarios en tiempo real y diferido, significó una novedad, no obstante, el hándicap a superar fue que se aportó una infraestructura en la que los participantes siguieron los contenidos del congreso e interactuaban, con el moderador y entre sí, generando un debate propio y accesible entre ellos, pero no interactuaron con los ponentes del congreso, ni la información que ellos produjeron trascendió otros grupos o comunidades.

La experiencia del Congreso CUICIID 2012, que ahora se estudia, significa una nueva modalidad de mediación tecnológica de las TIC a través de una red social que además de servir de canal para la transmisión de vídeo en multiconferencia establece una nueva forma de difundir el trabajo científico mediante un conjunto de servicios o Cloud Computing, en los que Google Plus se destaca ofreciendo SaaS y PaaS ${ }^{5}$. El Cloud Computing, nube de servicios o servicio en la nube, es "un nuevo paradigma computacional que permite a los usuarios utilizar una infraestructura a través de la red, como un servicio proporcionado por un proveedor". (ORSI, 2010:10).

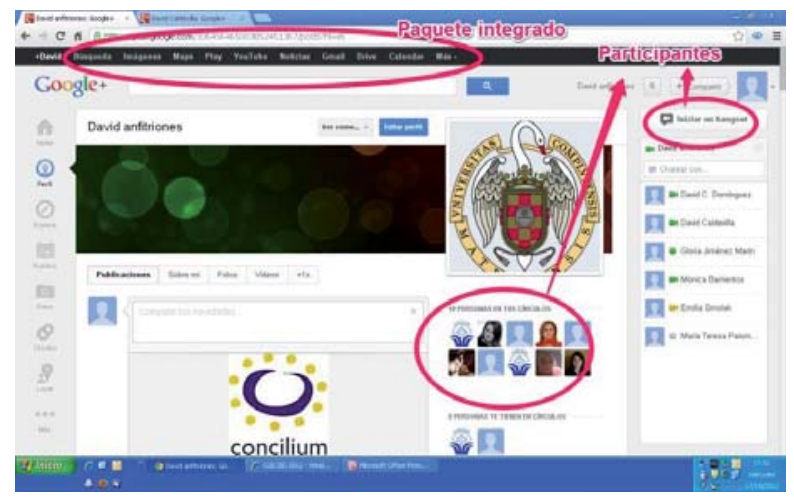

Figure 2. Perfil del Director del Congreso, que da cuenta de la estructura integrada de Google Plus, la personalización que permite la interfaz mediante los logos de la UCM y del grupo de investigación CONCILIUM, así como los participantes antes de ser invitados al "Hangout" o "quedada", modalidad que permite la multiconferencia.

Google ofrece un paquete integrado de servicios en la nube entre los que se incluyen el Gmail (mensajería), Google Docs (construcción colaborativa de documentos), Google Calendar (planificación mediante calendario), Picassa (gestión de fotografías); Geolocalización, Google Groups, Google Drive (almacenamiento de todo tipo de archivos). Google Plus es una red social que integra todos estos servicios y gestiona la información a partir de círculos que permiten organizar a los usuarios por perfiles

${ }^{5}$ SaaS: Software as a service / el Software como servicio. PaaS: Platform as a Service / Plataforma como servicio. 
y acceder en sistema multiplataforma, es decir, a través de un ordenador, un Smartphone o una Tablet.

\section{CUICIID virtual y en línea}

El Congreso Universitario Internacional sobre la Comunicación en la Profesión y en la Universidad de Hoy: Contenidos, Investigación, Innovación y Docencia (CUICIID 2012 Virtual y En Línea), se realizó utilizando como canal de comunicación, de la organización con los asistentes, su propia web ${ }^{6} \mathrm{y}$, como mediador tecnológico para la realización del Congreso la red social Google Plus, los días 17 y 18 de octubre de 2012. La idea surgió, según relata el director del Congreso, David Caldevilla Domínguez, buscando llegar de manera gratuita a una audiencia ávida del intercambio científico, que se encontraba dispersa en España y algunos países europeos, pero principalmente en América del Sur?

El congreso pretendía ser "un referente dentro de la necesaria simbiosis entre el mundo profesional y el docente, haciendo especial hincapié en la innovación docente, las nuevas líneas en investigación y la transmisión de nuevos contenidos a través de las TIC", por lo cual, la convocatoria se dirigió a investigadores, docentes y profesionales y planteó cuatro áreas temáticas mediante la modalidad de mesas de trabajo: A. Área de Innovación. B. Área de Investigación. C. Área de Docencia. D. Área de Miscelánea: para investigaciones con alto interés científico y/o con carácter multidisciplinar que no se ajuste a las anteriores ${ }^{8}$. La participación en el congreso requería que todos los participantes tuvieran una cuenta de Gmail para acceder a la interfaz de Google Plus, además, de contar con un ancho de banda mínimo de $1 \mathrm{Mb}$ de bajada y de subida, en el caso de conferencias 1 a 1 ; y de $2 \mathrm{Mb}$ de bajada y 1 de subida para 2 o más personas. Cámara de vídeo y micrófono, así como instalar el plugin de vídeo de Google.

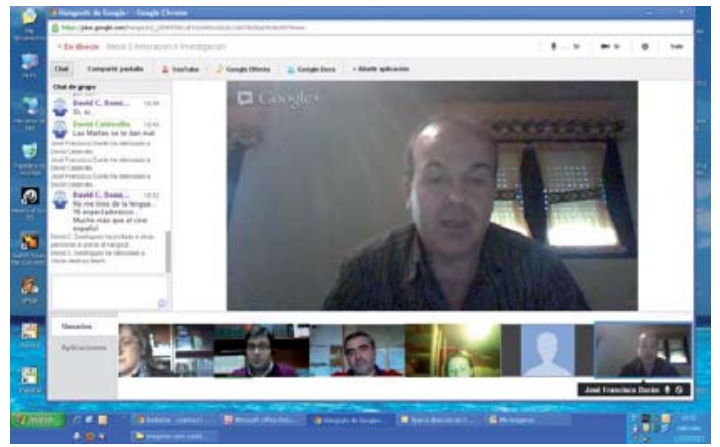

Figure 3. Captura de pantalla de la sesión del 18 de octubre de 2013. Mesa 2 del CUICIID "Innovación e Investigación”, conformada por 6 ponentes y un moderador, localizados en Madrid, Toledo, Sevilla y Polonia.

6 Website del Congreso: http://www.seeci.net/cuiciid/

7 Entrevista con el director del Congreso. Madrid, Febrero de 2013.

8 Material interno de la organización, difundido parcialmente en la web del congreso y vía email. Las mesas de trabajo se pueden consultar en la web del congreso en "Comunicaciones por mesas". 
Para su desarrollo, la organización envió un tutorial a los participantes y brindó asistencia técnica in situ. Se presentó, a la manera de "novedades" una doble propuesta de actuación congresual-No presencial: a) Modalidad Virtual o En Línea, la cual contemplaba la posibilidad de participar-voluntariamente- en videoconferencias múltiples (máximo de 8 ponentes más un moderador de mesa), el día y hora correspondiente a cada participante según la mesa temática. b) Modalidad Web No Presencial, basada en descargas sobre las aportaciones, es decir, colgar la ponencia en la web oficial del congreso para ser consultada libremente y comentada en bitácoras generadoras de debates por cada mesa temática de trabajo. Los trabajos podían colgarse como archivos .doc, que la organización convertiría a pdf; archivos de voz en .mp3; archivos de imagen en .avi.

La visibilidad de los trabajos y de la actividad de los participantes, se multiplicó gracias a la interacción de otros públicos, lo cual se logró de varias formas pero sobre todo por el vídeo que "fue un verdadero plus para el Congreso por la interacción e intimidad que generó además de la calidad y visibilidad que ofreció"9. En primer lugar, el Congreso se emitió en directo a través de You Tube, y en diferido en la web del Congreso. Una segunda experiencia surgió gracias a la técnica que, conectó la virtualidad con la presencialidad.

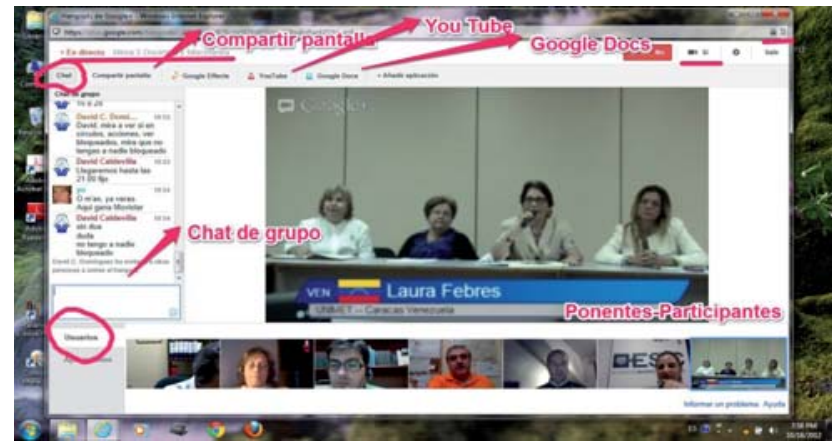

Figure 4. Mesa Miscelánea. 18 de octubre de 2012. La mesa interconectó ponentes de España, Colombia y Venezuela

La experiencia congresual se vio favorecida por la multiconferencia, cuando el equipo técnico de la universidad conectó la señal de la cámara con el ordenador y una pantalla gigante en un aula, desde la cual, simultáneamente las ponentes participaban en el Congreso y, contaban con un público presencial de aproximadamente 25 estudiantes de la Universidad Metropolitana de Venezuela, UNIMET. María Elena del Valle, ponente-participante destacó la visibilidad como el plus más importante del Congreso. También se utilizaron recursos como el chat, para intercomunicar los ponentes de manera individual y/o colectiva. Se podía compartir la pantalla del escrito-

9 Conclusión recurrente de moderadores y participantes, entre los que se destacan: David Caldevilla (director del congreso y moderador), Juan Enrique Gonzálvez (moderador de 2 mesas), José Rodríguez Terceño (moderador 2 mesas), María Elena del Valle participante, Alhim Adonaí Vera, entre otros. 
rio, lo cual permitió presentar la ponencia a través de un Power Point, o crear una franja de identificación con los nombres y logos incluidos por cada ponente.

Al hablar del factor multiplicador, significa que se han generado nuevas experiencias congresuales en la nube por parte de estas participantes, abriendo camino en el uso de esta herramienta y haciendo que la UNIMET tome como referente el principio de disminución de costes en la difusión del evento. La multiconferencia fue destacada por otros participantes, como elemento diferenciador y, el hecho de que, además de visibilidad, se facilitó una verdadera actividad de intercambio colaborativo entre equipos de investigadores multiculturales (Polonia, Venezuela, EE.UU., Méjico, Colombia, Venezuela y diferentes autonomías de España) y multidisciplinares (Comunicación, Publicidad, Empresariales, Economía, Derecho y otras), el plus se expresa además en una difusión más amplia que, en últimas, facilita el acercamiento de la universidad y la ciencia a la sociedad.

El ingeniero Ricardo Ardila Vetrovec de UNIMET señaló que se han realizado múltiples experiencias de videoconferencias desde 2004, en las que la estabilidad se ha visto afectada de diversas formas, motivo por el que se ha pasado de trabajar con equipos profesionales, a decantarse por servicios de programas gratuitos como Skype, Facetime o Google Chat. Pero, afirmó, "con el "hangout" de Google Plus, se puede lograr un resultado cercano al de los equipos profesionales a muy bajo costo ya que la carga tecnológica recae sobre Google. Eso es el Cloud Computing"10. A su vez, destaca que tras la experiencia con Google Plus se recomendó repetir ésta para un evento de la universidad del que, ante la satisfacción de los participantes se va a mantener esta experiencia en el ámbito educativo: defensa de tesis, jornadas, seminarios, etc.

Como estrategia de difusión de los resultados de investigación el Congreso planteó "novedades" con el ánimo de divulgar y dar mayor visibilidad a los trabajos de sus 340 participantes. Se buscó así que la finalidad del congreso trascendiera la aceptación de un manuscrito y tras el debate se generaran varias publicaciones con intención de difusión y visibilidad en los canales convencionales de difusión. Otra cosa sería la estrategia individual que puede diseñar cada autor (poseedor de los derechos de autor), en la Web 2.0; la cual puede contemplar repositorios temáticos, alojamientos en blogs, o presentaciones, además de la difusión en redes sociales o la inclusión en abierto o dominio público en servicios como Google Libros o Europeana, entre otros.

En primer lugar, se publicarían los resúmenes de los trabajos aceptados previa revisión de pares ciegos, en formato CD-Rom. Como segunda opción se planteaba la publicación de la ponencia completa en libro de papel con ISBN, por cada mesa de trabajo, con un máximo de 12 autores por libro. Finalmente, una tercera opción, voluntaria, implicaba la realización de un nuevo artículo, tras el congreso y a partir de la ponencia, a publicarse en la revista "Estudios sobre el Mensaje Periodístico: Investigaciones", previa revisión de pares ciegos. Según se explicó ${ }^{11}$, éste número recibe artículos originales (sobre Docencia, Innovación, Investigación y Nuevas Tendencias

${ }_{10}$ Comentarios y aportaciones suministrados por escrito, vía email.

11 Documentación aportada al Comité Organizador del Congreso 
en el Marco del EEES), que deben ajustarse a los objetivos científicos y divulgativos de la revista y que, hayan sido aprobados, tras revisión, por el Comité Científico y Evaluador, y el del CUICIID 2012.

Las actas del congreso, pueden emitirse o editarse, pero en cualquier caso, "aportan información esencial tanto para el conocimiento de las investigaciones en curso como para el conocimiento actualizado y preciso de las distintas fases de las investigaciones" (Moreno-Torres, 1986, web). En el primer caso: emitir las actas configura un tipo de literatura gris de nueva generación, al ser producida en formato digital y de manera limitada, es decir, coincidiendo con el número de participantes en el congreso. Al no estar disponibles comercialmente, los resúmenes de presentación de trabajos al Congreso se difunden a través de sus actas, pero su visibilidad se limita a algunas bibliotecas y a los participantes del congreso.

Por el contrario, en el segundo caso, la posibilidad de editar el libro con las ponencias del Congreso, previamente revisadas y/o optativamente debatidas por sus autores, constituye una forma de brindar mayor visibilidad al trabajo científico ofreciendo un producto de literatura científica convencional, catalogable al tener ISBN y depósito legal y, presentado con la intención clara de difusión al ofrecerse en la opción de Impresión Bajo Demanda. Esta opción, ofrece, como es lógico, un mayor acercamiento a otras comunidades científicas y a la sociedad en general al estar disponible comercialmente y difundirse abiertamente. Nuevamente la tecnología hace posible una disminución de costes considerando que publicar un libro ya no requiere de grandes inversiones sino de nuevas opciones. De igual manera, la publicación en la revista EMP genera un nuevo artículo, motiva la producción de conocimiento y activa un nuevo proceso de revisión de pares que reta al autor a presentar distintos resultados derivados de su investigación. Se destaca aquí el hecho de que no existe duplicidad en la información que se genera (Actas como resúmenes, libro como ponencias revisadas y artículo revisado para revista), añadiendo el hecho de que se están utilizando formatos diferentes (CD- Rom, en soporte físico-digital, libro y revista, en soporte físico-papel).

\section{Conclusiones}

Si bien los congresos en general propenden por la difusión y socialización de productos, servicios, ideas, etc., los congresos científicos, en particular, constituyen eventos donde ciencia, tecnología, educación y comunicación convergen como elementos que permiten el acercamiento de los trabajos académicos y científicos al mundo empresarial y a la sociedad en general. El Congreso CUICIID 2012, modalidad virtual y en línea, propició la integración multidisciplinar e interinstitucional de sus asistentes, trasvasó las opciones de publicación creando estrategias que minimizaron la producción de la literatura gris y, generó publicaciones de literatura convencional, es decir, registradas bajo depósito legal, que coadyuvan a aumentar la visibilidad del trabajo de los investigadores y que, permiten que los resultados de investigaciones sean accesibles a públicos mayores al entrar éstos en el circuito comercial.

Los servicios Cloud Computing de Google Plus son, de momento, una herramienta útil para docentes e investigadores que en el caso de la realización del Congreso CUI- 
CIID, lograron acceder de forma sencilla, interactuar desde diversos países, replicar el modelo en otras esferas internacionales y aumentar la visibilidad del trabajo científico de los participantes. No obstante, la experiencia demostró que la apropiación de las TIC requiere todavía tiempo y esfuerzo. El Congreso, motivó e integró los 3 roles de los asistentes: autor, asistente y relación brindándoles interacciones y visibilidad en y con la web 2.0; promovió el acceso de los ciudadanos al conocimiento científico y fomentó buenas prácticas de una aplicación tecnológica de libre acceso. A su vez, se promovió la alfabetización tecnológica y la cultura científica, y se redujeron los costes en la realización de un evento de carácter internacional, colaborativo y multicultural.

Finalmente reiterar que el "triángulo de conocimiento": educación, ciencia, innovación fusionado con el aprovechamiento de las TIC, el aprendizaje permanente y las competencias estructura un nuevo constructo socioeducativo en el que ciudadanos científicamente alfabetizados serán "menos vulnerables a la propaganda, al consumismo, a los discursos dogmáticos, y más críticos y capaces de tomar decisiones fundadas" (COSTA, 2009, web) en aras de consolidar una alfabetización científica, tecnológica y mediática, edificadora de nuevos ciudadanos en la actual Sociedad del Conocimiento.

\section{Referencias bibliográficas}

AA.VV. (2003): "Comunicación de la Comisión: El papel de las universidades en la Europa del Conocimiento". Comisión de las Comunidades Europeas. Bruselas 05.02.2003. http://www.crue.org/export/sites/Crue/procbolonia/documentos/antecedentes/7._El_papel_de_las_universidades.pdf.

AA.VV. (2006): "Recomendación del Parlamento Europeo y del Consejo de 18 de diciembre de 2006 sobre las competencias clave para el aprendizaje permanente (2006/962/CE)". Diario oficial de la Unión Europea L 394/10. http://www.mcu.es/cine/docs/Novedades/Recomendacion_Parlamento_Europeo_Consejo_Aprendizaje_permanente.pdf

BUELA-CASAL, Gualberto; BERMÚDEZ, M ${ }^{\text {a }}$ Paz; SIERRA, Juan Carlos; QUEVEDO-BLASCO, Raúl; CASTRO, Ángel; y GUILLÉN-RIQUELME, Alejandro (2012): "Ranking de 2011 en producción y productividad en investigación de las universidades públicas españolas", en: Psicothema: http://www.psicothema.com/psicothema.asp?id=4046 [fecha de consulta: 1 de febrero de 2013]

COSTA, Joan (2009): "La Unión Europea y la Sociedad del Conocimiento. La comunicación pública de la ciencia". Revista Telos, $\mathrm{n}^{\circ}$ 57, segunda época. http://sociedadinformacion.fundacion.telefonica.com/telos/perspectivaimprimible.asp@ida rticulo $=1 \&$ rev $=57$. htm

HURD, Julie M (2000): “The Transformation of Scientific Communication: A Model for 2020". Journal of the American Society for Information Science. 15(14): 12791283: http://www.ou.edu/ap/lis5703/sessions/hurd.pdf.

NEGROPONTE, Nicholas (1995): El mundo digital. Barcelona, Ediciones B. 
ORSI (2010): "Cloud Computing. La Tecnología como Servicio". ORSI. Observatorio Regional de la Sociedad de la Información de Castilla y León. Disponible en: www.orsi.jcyl.es

RUIZ-SANTOS, Candelaria y MEROÑO-CERDÁN, Ángel Luis. (2007): “Utilidad de los congresos científicos en la difusión del conocimiento: percepción del investigador español en Economía de la Empresa, en Ciencia y Técnica Administrativa. http://www.cyta.com.ar/ta0602/v6n2a1.htm.

SANTOS, Clara Janneth (2012): "Las nuevas tecnologías y la difusión del conocimiento científico: Los congresos virtuales o e-congresos", en BERNARD, Estela: Labores docentes e investigadoras en el EEES. Barcelona, Visión Libros, pp. 269292.

TORRES-SALINAS, Daniel y DELGADO-LÓPEZ-CÓZAR, Emilio (2009): "Estrategia para mejorar la difusión de los resultados de investigación con la Web 2.0", en El profesional de la información, vol.18, $\mathrm{n}^{\circ}$ 5, septiembre-octubre.

\section{Clara Janneth SANTOS MARTÍNEZ}

Universidad Autónoma de Bucaramanga. Colombia. Facultad de Educación.

Doctora en Ciencias de la Información por la Universidad Complutense de Madrid, 1999, periodista e investigadora. Docente Virtual de la Especialización en Educación con Incorporación de NTICS.

janneths@hotmail.com 